\title{
Potential Prognostic Molecular Signatures in a Preclinical Model of Melanoma
}

\author{
SOTIRIOS P. FORTIS, ELEFTHERIA A. ANASTASOPOULOU, IOANNIS F. VOUTSAS, \\ CONSTANTIN N. BAXEVANIS, SONIA A. PEREZ and LOUISA G. MAHAIRA
}

Cancer Immunology and Immunotherapy Center, Saint Savvas Cancer Hospital, Athens, Greece

\begin{abstract}
Numerous studies have revealed a variety of pathways involved in the development of melanoma, however, the molecular and genetic divergence of underlying mechanisms remain vague. In a mouse model, we studied the expression pattern of insulin-like growth factor 2 mRNAbinding protein 1 (Igf 2bpl) and target genes microphthalmiaassociated transcription factor (Mitf), v-myc avian myelocytomatosis viral oncogene homolog (Myc), B-cell lymphoma 2 (Bcl2), prothymosin alpha (Ptma) and melan- $A$ (Mlana) in relation to tumor-growth characteristics. The in vivo expression of the aforementioned genes was assessed by quantitative Real Time-Polymerase Chain Reaction (RT-PCR) in tumors established by B16-F1-derived clones. Gene expression was correlated with tumor growth characteristics. Simultaneous expression of elevated levels of Myc, Igf $2 \mathrm{bpl}$, Ptma and Mitf characterizes tumors with a more aggressive phenotype. Our findings introduce a tumor-specific molecular signature possibly associated with melanoma heterogeneity. The concomitant overexpression of key molecules such as IGF2BP1, PTMA, MYC and MITF could serve as prognostic or predictive marker.
\end{abstract}

Melanoma is a very heterogenic cancer with great in vivo plasticity (1). Although the diversity of melanomas is known, the underlying molecular and genetic differences remain obscure (2). Thus, identifying intra-tumoral molecular signatures might introduce novel biomarkers of prognostic significance. A number of studies have unveiled a variety of molecules and several distinct pathways involved in the onset of melanoma. Some focused on the multifaceted and complicated role of wingless-related integration site $(\mathrm{Wnt}) / \beta$ catenin pathway $(3,4)$. However, other studies have also

Correspondence to: Louisa G. Mahaira Ph.D., Cancer Immunology and Immunotherapy Center, Saint Savvas Cancer Hospital, 171 Alexandras Avenue, 11522, Athens, Greece. E-mail: mahaira@ciic.gr

Key Words: Melanoma, Igf2bp1, Ptma, $\beta$-catenin/Wnt pathway, molecular signatures. pinpointed microphthalmia-associated transcription factor (MITF) and insulin-like growth factor 2 mRNA-binding protein 1 (IGF2BP1) as drivers in melanoma initiation and progression.

MITF is a major transcription factor, dynamically transcribed by $\beta$-catenin complexes (5), active in normal melanocytes and malignant melanoma cells (6). Its deregulated expression has been described as a causal event in melanomas, resulting from gene amplification (7), as well as, from the ectopic expression of the RNA binding protein IGF2BP1 (8). As an RNA-binding protein, IGF2BP1 exerts its mode of action via regulating the intracellular levels of its targets. Among them, besides MITF, there are many notorious oncogenes such as v-myc avian myelocytomatosis viral oncogene homolog $(M Y C)$, glioma-associated oncogene family zinc finger $1(G L I 1)$, insulin-like growth factor 2 (IGF2), Kirsten rat sarcoma viral oncogene homolog (KRAS), multidrug resistance protein $1(M D R-1)$, beta-transducin repeat containing E3 ubiquitin protein ligase pseudogene 1 (BTRCPI) and homing cell adhesion molecule (CD44) (9). It has been reported that overexpression of IGF2BP1 results in upregulation of one or more of these target genes, depending on the specific type of tissue (10). Although its expression is absent or scarce in normal adult tissues, it is detected in almost all types of cancers (9). It is common knowledge that heterogeneity from a single cell level to the whole solid tumor is mainly the outcome of the well-orchestrated differential expression of a multitude of genes, carved under the pressure of the tumor microenvironment.

Given the established role of IGF2BPI and MITF in melanoma, here we investigated the expression pattern of these genes, along with the associated molecules $M y c$, B-cell lymphoma 2 (Bcl2), prothymosin alpha (Ptma) and melan-A (Mlana), in relation to growth characteristics, in the context of melanoma heterogeneity. Although the Achilles' heel of all types of melanomas is very difficult to identify, novel combinations of gene expression patterns, often described separately to be involved in melanoma, might better serve as a more representative molecular signature for melanoma characterization and prediction. 
Table I. Sequences of primers for the gene expression analysis.

\begin{tabular}{|c|c|c|c|c|c|}
\hline Gene & Protein & Forward primer 5 ' -3 ' & Reverse primer $5^{\prime}-3$ ' & $\mathrm{Tm}$ & Reference (no) \\
\hline Gapdh & $\begin{array}{l}\text { Glyceraldehyde 3-phosphate } \\
\text { dehydrogenase }\end{array}$ & GTGTTCCTACCCCCAATGTG & GTCATTGAGAGCAATGCCAG & 59 & $(26)$ \\
\hline Igf $2 b p 1$ & $\begin{array}{l}\text { Insulin-like growth factor } 2 \\
\text { mRNA-binding protein } 1\end{array}$ & GGCTGCTCCCTATAGCTCCT & CTGGTGGTGCAATCTTGATG & 59 & (26) \\
\hline$B c l 2$ & B-cell lymphoma 2 & CTGTGGATGACTGAGTACCT & AGCCAGGAGAAATCAAACAG & 60 & M\&M section \\
\hline Myc & $\begin{array}{l}\text { v-myc avian myelocytomatosis } \\
\text { viral oncogene homolog }\end{array}$ & AGCGACTCTGAAGAAGAGCAA & GTTGTGCTGGTGAGTGGAGA & 62 & (26) \\
\hline Ptma & Prothymosin alpha & TTGTGGAGGAGGCAGAGAAT & TTGGTGTCCACATCGTCATC & 62 & M\&M section \\
\hline Mlana & Melan-A & ATGTGAGAGCCCTGATCACC & TAAGGTGGCGGTGAAGAGAG & 59 & M\&M section \\
\hline Mitf & $\begin{array}{l}\text { Microphthalmia-associated } \\
\text { transcription factor }\end{array}$ & GTGCAGACCCACCTGGAAAAC & AGTTAAGAGTGAGCATAGCCATAG & 60 & $(27)$ \\
\hline
\end{tabular}

\section{Materials and Methods}

Cell lines and generation of clones. B16-F1 melanoma murine cell line (American Type Culture Collection, Wesel, Germany) Cultures maintained in logarithmic phase of growth and sub-cultured using Trypsin $0.25 \%$-ethylene-diaminetetraacetic acid solution (Life Technologies, Waltham, MA, USA). Clones were generated applying the limiting dilution assay, by seeding 1 cell/well in 96-well plates Dulbecco's Modified Eagle Medium (Life Technologies, Waltham, MA, USA). Media were replenished every two days. The total number of clones was 22 , in compliance with the estimated numbers by the likelihood equations of this assay.

Proliferation assay. The proliferative rate of each cell line was estimated by thymidine incorporation. Briefly, cells were seeded at 5,000 cells/well in 96-well flat bottomed plates (Greiner Bio-One $\mathrm{GmbH}$, Kremsmünster, Austria) and pulsed with $1 \mu \mathrm{Ci} /$ well [methyl- ${ }^{3} \mathrm{H}$ ] thymidine (specific activity: $53.0 \mathrm{Ci} / \mathrm{mmol}, 1 \mathrm{mCi} / \mathrm{ml}$; Amersham, GE Healthcare, Amersham, UK) for additional $18 \mathrm{~h}$. Cells were harvested $48 \mathrm{~h}$ post seeding and counted on a liquid scintillation counter (Wallace Trilux, No. 1450 Microbeta; PerkinElmer Life Sciences, Waltham, MA, USA).

Animals. All protocols were in compliance with the Greek and European regulations on Animal Welfare and with Public Health Service recommendations. Female C57BL/6 mice, aged 6-8 weeks old (Hellenic Pasteur Institute, Athens, Greece) were randomly assigned (at least five per clone or line) to receive $5 \times 10^{5}$ cells in $100 \mu \mathrm{l}$ Phosphate Buffered Saline (Biochrom GmbH, Berlin, Germany), by subcutaneous injection in their flank (single flank/mouse). Tumor growth was monitored daily and growth measurement is presented as the orthogonal mean: $(\mathrm{d} 1+\mathrm{d} 2) / 2$. All animals were sacrificed by $\mathrm{CO}_{2}$ inhalation, on the 16th day post inoculation. Tumors were excised, weighed and dissociated mechanically. Single-cell suspensions were used to assess antigen expression by flow cytometric analysis and gene expression by quantitative real-time polymerase chain reaction (qRTPCR). In order to facilitate our combinatorial analysis, all samples were divided for each parameter (growth and gene expression) into two groups based on the median value of this parameter in all tumors (irrespective of the clone of origin) namely high and low.

Flow cytometry. Cells were washed and stained as previously described (11). In detail, cells were stained with the monoclonal antibodies anti-CD45 (leukocyte common antigen (LCA)) (clone 30F11; eBioscience, Cheshire,UK) and anti-CD44 (clone IM7, eBioscience), fixed and analyzed on a BD FACS Canto II instrument (BD Biosciences, Milano, Italy) using DIVA 6.0 software (BD Biosciences, Milano, Italy).

$R N A$ extraction and $q R T-P C R$. Total RNA extraction was performed using Nucleospin RNA II kit (Macherey-Nagel GmbH\&Co, Düren, Germany) according to the manufacturer's instructions. Total RNA (1 $\mu \mathrm{g})$ was reverse transcribed using PrimeScript Reverse Transcriptase (TaKaRa, Bio.Inc, Shiga, Japan) and random hexamer oligonucleotides (Invitrogen, Life Technologies). qRT-PCR was performed on a RotorGene 3000 instrument (Corbett Research, Life Technologies). cDNA solution (200 ng) KAPA SybrFast qPCR kit (KapaBiosystems, London, UK) and $200 \mathrm{nMol}$ of specific primers (VBC Biotech Service, Wien, Austria) were used in a $20 \mathrm{ml}$ reaction. Sequences of primers are listed in Table I. Primers for Ptma, Bcl2 and Mlana were designed via Primer3 software v.0.4.0 (http://bioinfo.ut.ee/primer3-0.4.0/primer3/). In order to confirm amplification specificity, melting curve analysis was performed and the $\Delta \Delta \mathrm{Ct}$ method was applied for gene expression quantification. Glyceraldehyde 3-phosphate dehydrogenase (Gapdh) was used for internal normalization and the B16-F1 cell line as reference sample.

Statistical analysis. Statistical analyses were performed by GraphPad Prism v.5.0 (GraphPad software, La Jolla, USA), using Spearman's correlation with $95 \%$ confidence interval, Fisher's exact test and Mann-Whitney $t$-test, accordingly. $p$-Values of less than 0.05 were considered statistically significant.

\section{Results}

Generation of a preclinical model reflecting melanoma heterogeneity and differential expression of Igf $2 \mathrm{bpl}$ in vitro and in vivo. In order to create a preclinical melanoma model reflecting melanoma diversity, we used B16-F1-derived clones exhibiting different in vitro proliferation rates (Figure 1a), cell morphology, anchorage independence, melanin production and gene expression pattern to generate tumors in C57BL/6 mice (data not shown). The differential profile of the clones noted in vitro, still existed in vivo, as was confirmed by the tumor growth rate (Figure 1a) and gene expression analysis (data not shown). 

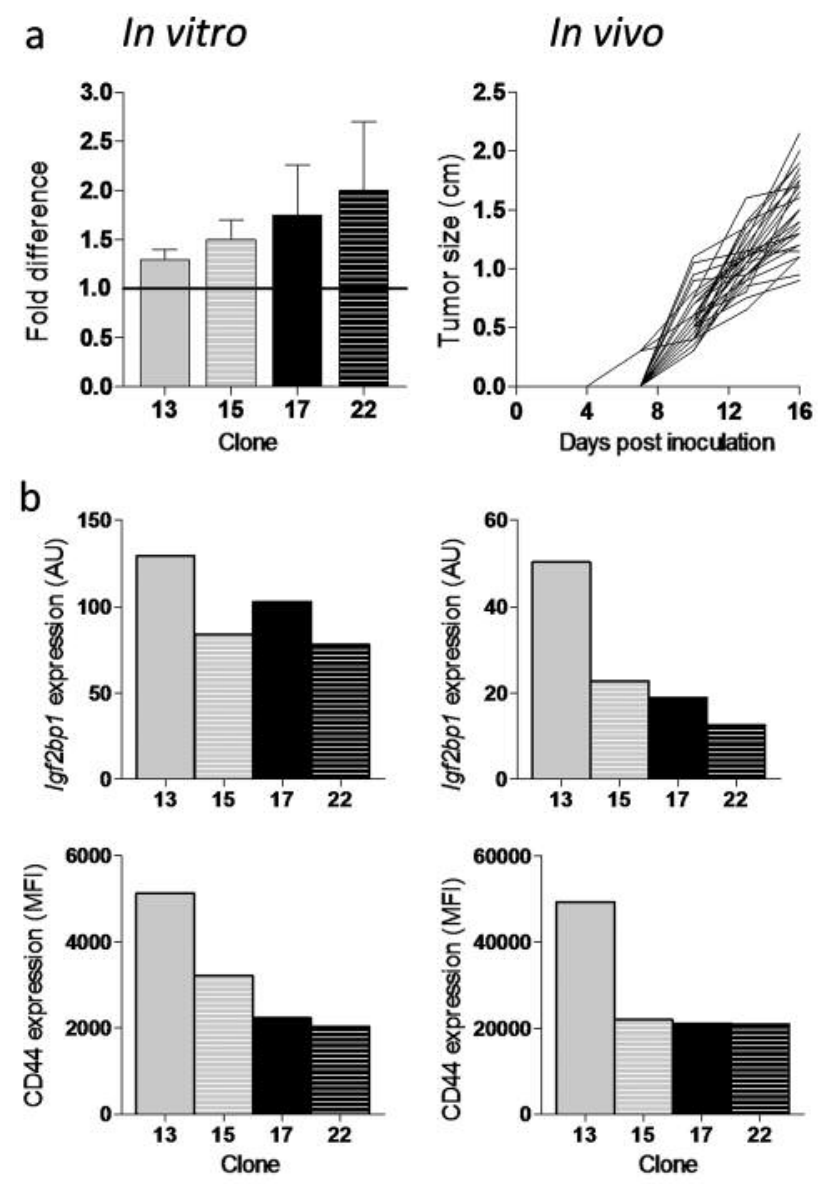

Figure 1. a: Left: In vitro proliferation rate of B16-derived clones $13,15,17$ and 22. Bars represent the fold difference of each clone in relation to the maternal cell line $(B 160-F 1=1.0)$. Right: In vivo proliferation, where each line represents growth of an individual tumor. $b$ : In vitro and in vivo expression of insulin-like growth factor 2 mRNAbinding protein 1 (Igf2bp1) mRNA (median value of $A U$ ) and homing cell adhesion molecule (CD44) antigen in clones 13 (50.21, range:23.49-72.20), 15 (22.76, range:12.07-111.7), 17 (18.95, range:0.92-27.93) and 22 (12.5, range:9.21-36), ( $n=5 /$ group). AU: Arbitrary Units; MFI: mean Fluorescence Intensity.

CD44 and Igf $2 b p l$ levels of expression were assessed on the 16th day (Figure 1b). Considering the role of IGF2BP1 in melanoma, we chose clones 13 and 22, which exhibited the most significant difference in in vivo expression of $I g f 2 b p l$ $(p=0.0317)$, as well as of antigen CD44 (Figure 1b). Our group (10) and others have shown that high levels of IGF2BP1 and the presence of CD44 molecule are related to more aggressive melanoma phenotype (11), characterize mesenchymal cells and are linked post-transcriptionally (12). Tumors generated by these clones had similar growth (gr), since their median mass did not differ significantly ( $p=0.3651$; Figure $2 \mathrm{a}$ ). However, the diameter of clone 22 tumors was significantly greater than
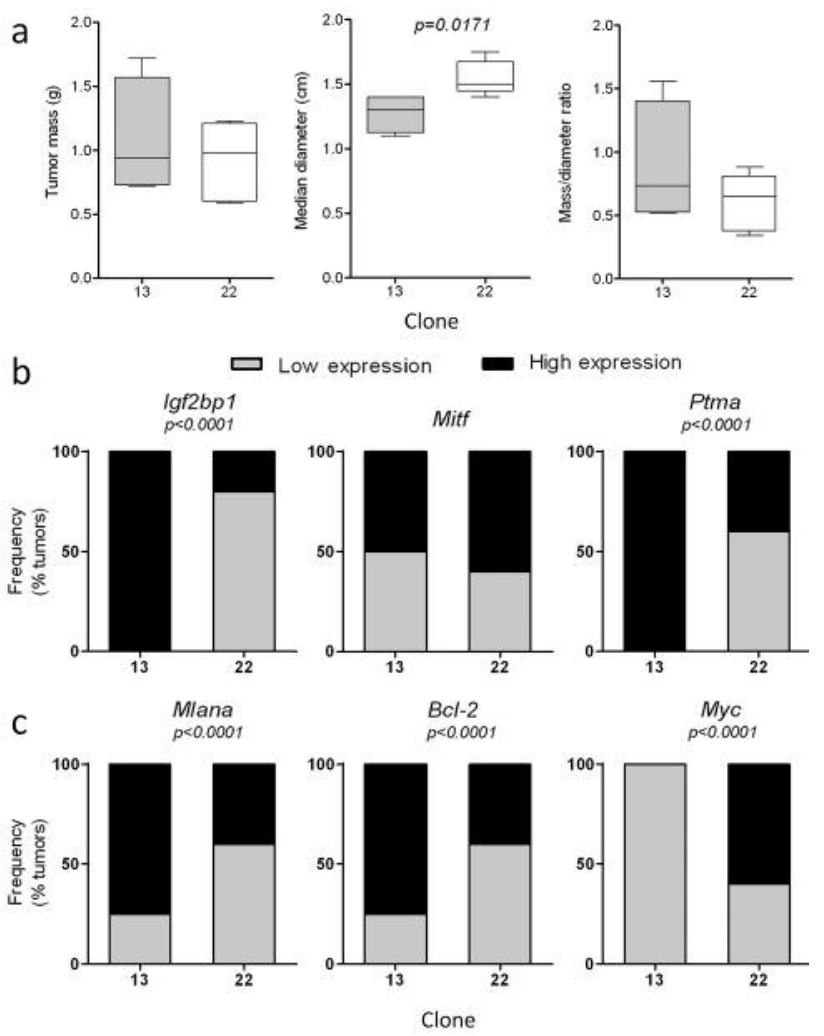

Figure 2. Growth characteristics and gene expression in tumors derived from clones 13 and 22. a: Boxplots represent median values of mass, diameter, and mass to diameter ratio of tumors derived from clones 13 $(0.94 \mathrm{gr}, 1.3 \mathrm{~cm}, 0.74$, respectively) and $22(0.98 \mathrm{gr}, 1.5 \mathrm{~cm}, 0.65$, respectively) ( $n=5 /$ group). $b$ : Bars represent the frequency of tumors with high or low level of insulin-like growth factor $2 \mathrm{mRNA}$-binding protein 1 (Igf2bpl), prothymocin alpha (Ptma), microphthalmiaassociated transcription factor (Mitf), $v$-myc avian myelocytomatosis viral oncogene homolog (Myc), melan-A (Mlana) and B-cell lymphoma 2 (Bcl2) expression ( $n=5 /$ group).

that of clone 13 tumors ( $p=0.0171$; Figure 2a), suggesting a different pattern of in vivo growth, possibly implying a preferential growth in depth (reflected by mass/diameter ratio) for clone 13 .

Gene expression pattern differs between clones with different Igf 2 bpl expression. Clones 13 and 22 were analyzed for their gene expression pattern. Figure $2 b$ presents the percentages of tumors exhibiting high or low expression of each gene studied. Interestingly, Igf2bpl, Ptma, Bcl2 and Mlana exhibited a common pattern of expression. The majority of clone 13derived tumors showed high levels of expression of these genes compared to clone 22 . All clone 13-derived tumors $(100 \%)$ expressed high levels of Igf $2 b p I$ and Ptma but low levels of $M y c$. It is noteworthy that clone 22-derived tumors exhibited the opposite pattern of gene expression. 

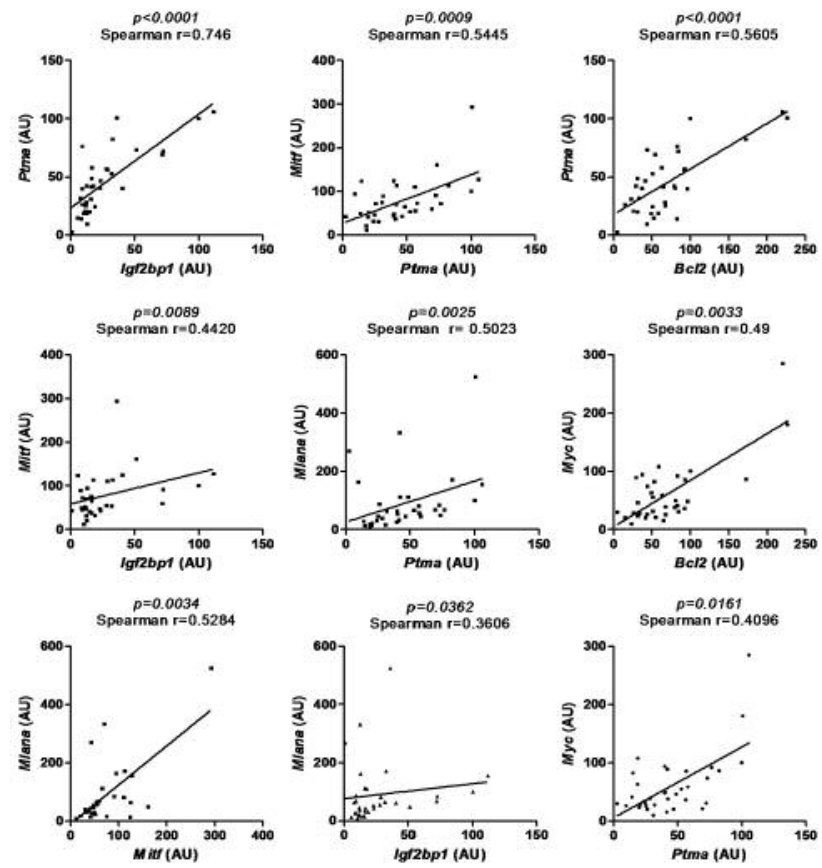

Figure 3. Correlations of gene expression. Graphs present correlations in gene expression (AU) of all genes studied. Igf $2 \mathrm{bpl}$ : insulin-like growth factor 2 mRNA-binding protein 1; Ptma: prothymocin alpha; Myc: v-myc avian myelocytomatosis viral oncogene homolog; Mlana: melan-A; Bcl2: B-cell lymphoma 2; Mitf: microphthalmia-associated transcription factor.

Potential correlations in gene expression. Figure 3 depicts only the genes with positive correlations that were statistically significant. Correlations of Myc with Ptma $(p=0.0161)$ and Bcl2 ( $p=0.0033)$, and Mitf with Mlana $(p=0.0013)$ and Igf $2 b p I$ $(p=0.0089)$ were in line with previous studies regarding control of gene expression (13-15). However, strong positive correlations were also observed for Ptma with Igf $2 \mathrm{bpl}$ $(p<0.0001)$, Mitf $(p=0.0009)$ and Bcl2 $(p<0.0001)$, as well as Mlana $(p=0.0025)$. Moreover, Mlana weakly correlated with Igf2bpl $(p=0.0362)$.

Combinatorial analysis of gene expression in relation to tumor growth reveals distinct molecular patterns. Analysis of expression of single genes in relation to growth characteristics showed no significant correlation (data not shown). Therefore, we sought to test whether the concomitant expression of two or more genes correlated with similar in vivo tumor growth characteristics. Tumors generated by all clones were divided in sub-categories, based on the level of simultaneous expression of two genes of interest each time. The group with high levels was compared to the group with low levels, regarding their mass (Figure 4a), diameter (Figure 4b) and mass/diameter (Figure 4c). Interestingly, the analysis revealed that high expression of Igf $2 b p l$ with Ptma, Myc with Ptma or $c-M y c$ with
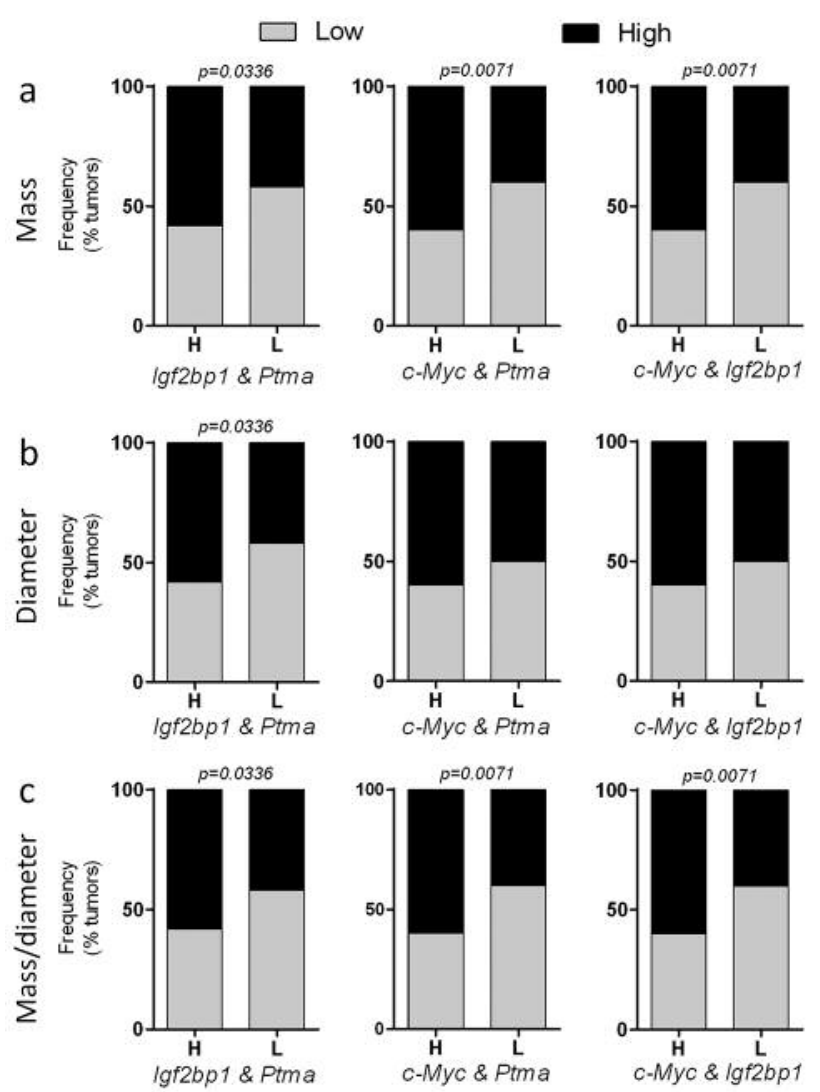

Figure 4. Tumor growth analyzed in relation to gene co-expression. Bars represent the frequency of tumors with high $(H)$ or low $(L)$ co-expression of both insulin-like growth factor 2 mRNA-binding protein 1 (Igf2bpl) and prothymocin alpha (Ptma) $(n=14), v$-myc avian myelocytomatosis viral oncogene homolog (Myc) and Ptma $(n=10)$, and Igf2bpl and Myc $(n=10)$ in relation to high or low mass $(a)$, diameter $(b)$ or mass to diameter ratio $(c)$.

Igfbpl correlated with high tumor mass and mass/diameter. Given the correlation for gene expression described previously, we decided to investigate the concomitant expression of three or more genes. We observed that co-expression of high levels of $M y c$, Igf $2 b p l$ and Ptma had an even stronger correlation with high tumor mass (Figure 5a) and mass/diameter (Figure 5c), which was maintained after including Mitf.

The effect of the expression pattern of Igf $2 b p l, M y c$ and Ptma on tumor growth was verified following analysis of tumors derived from clones with opposite reverse growth characteristics (clone 15 and 17 data not shown). Hence, expression of Igf $2 b p l, M y c$ and Ptma might serve as a possible molecular signature underlying melanoma in vivo growth rate.

\section{Discussion}

The results presented herein strongly suggest that concomitant activation of Igf2bpl, Myc, Ptma and Mitf are associated with 

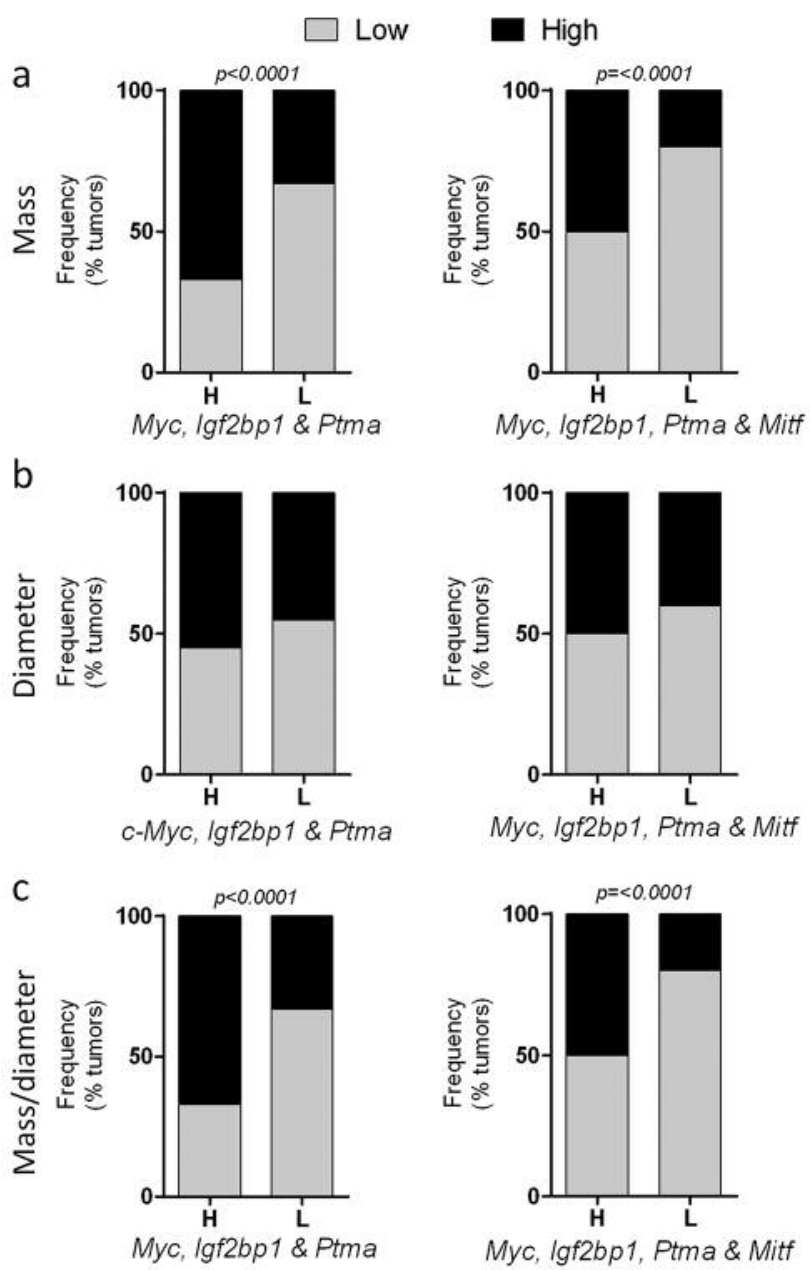

Figure 5. Combinatorial analysis of gene expression. Bars represent the frequency of tumors with high $(H)$ or low $(L)$ co-expression of insulinlike growth factor 2 mRNA-binding protein 1 (Igf2bp1), prothymocin alpha (Ptma) and v-myc avian myelocytomatosis viral oncogene homolog (Myc), (n=9), and of Igf2bp1, Ptma, Myc and microphthalmiaassociated transcription factor (Mitf), $(n=6)$ in relation to tumor mass $(a)$, diameter $(b)$ and mass/diameter ratio (c).

a more aggressive melanoma phenotype in our preclinical model. More specifically, our findings indicate that augmented co-expression of these genes characterizes melanoma tumors with increased in vivo growth rate.

Given the fact that high levels of Igf $2 b p 1$ and CD44 have been correlated with metastatic potential and aggressiveness in melanoma $(11,13)$, we decided to investigate the expression pattern of Igf2bpl, Myc, Ptma, Mitf, Mlana and Bcl2 in tumors with high or Iow expression of these genes. Based on our data, high levels of Igf $2 \mathrm{bpl}$, Ptma, Bcl2 along with Melan-A, an already established marker in melanoma characterization (16, 17), are associated with tumor of small diameter but high mass, implying melanoma invasiveness (18).
Previous reports have described the selected genes studied here as associated players in the $\mathrm{Wnt} / \beta$-catenin pathway, which is deregulated in most cases of melanoma (4). Mitf participates in a feed-back loop regulatory mechanism with $\beta$-catenin (19) and may alter its transcriptional activity (5). Additionally, Igf $2 \mathrm{bpl}$ and Ptma are direct targets in the $\beta$-catenin pathway $(20,21)$. Moreover, Igf2bp1 post-transcriptionally regulates Mitf and $M y c$, whose transcription is in turn controlled by $\beta$ catenin (22). These interactions dictate the high complexity of involvement of the $\mathrm{Wnt} / \beta$-catenin pathway in melanoma tumorigenesis. In line with this, we studied the above mentioned genes aiming to assign a specific expression pattern to differential melanoma growth rates.

Our analysis revealed significant correlations in gene expression, particularly for Ptma with $B c l 2, M y c$ and $I g f 2 b p l$. Correlation of Ptma and $B c l 2$ is anticipated given the fact that PTMA has been described as an anti-apoptotic molecule (23). A previous study found elevated PTMA correlated to increased Myc level in human colon carcinomna and was further associated with multiple established clinicopatho-logical factors as well as the clinical outcome (24). Notably, the significant correlation of Igf $2 b p l$ and Ptma, reported here for the first time to our knowledge, could be explained either by the fact that both these genes are affected by $\beta$-catenin in a parallel way, or by the hypothesis that Igf2bpl may bind to Ptma mRNA positively regulating its pattern of expression. This observation should be further investigated by gene silencing or analyses to elucidate the possibility that Ptma is another target of Igf $2 \mathrm{bpl}$.

Given the limited number of reports regarding concomitant expression of Igf $2 \mathrm{bpl}$ and Ptma in cancer, we studied their association with already described molecules in our effort to indentify a prognostic signature in melanoma. In particular, high co-expression of Igf $2 \mathrm{bpl}$, Ptma and $M y c$ characterizes tumors with high mass and high mass/diameter but not tumors with large diameter and low mass/diameter ratio. When Mitf, the major melanoma regulator, is added to the signature, this correlation persists, even though the remaining sample size is very small. Noteworthy, the results of the current study should be considered only as hypothesis generating, due to the large intra-group variation, possibly explained by melanoma plasticity (25), along with the limited number of animals analyzed.

Our findings suggest that the role of $\mathrm{Wnt} / \beta$-catenin pathwayrelated molecules in melanoma is not only qualitative but also quantitative. We propose that the concomitant overexpression of key molecules such as Igf2bpl, Ptma, Myc and Mitf, although perhaps not solely driven by the Wnt/ $\beta$-catenin pathway, may better reflect the aggressiveness of melanoma phenotype. Thus, this molecular signature could serve as prognostic or predictive marker for melanoma, adding more arrows to our quiver against this disease. Finally, extensive studies in primary human melanoma tissues, from patients with available follow-up data, are required in order to validate the prognostic significance of this signature. 


\section{References}

1 Roesch A, Paschen A, Landsberg J, Helfrich I, Becker JC and Schadendorf D: Phenotypic tumour cell plasticity as a resistance mechanism and therapeutic target in melanoma. Eur J Cancer 59: 109-112, 2016.

2 Harbst K, Lauss M, Cirenajwis H, Winter C, Howlin J, Torngren T, Kvist A, Nodin B, Olsson E, Hakkinen J, Jirstrom K, Staaf J, Lundgren L, Olsson H, Ingvar C, Gruvberger-Saal SK, Saal LH and Jonsson G: Molecular and genetic diversity in the metastatic process of melanoma. J Pathol 233(1): 39-50, 2014.

3 Moon RT, Kohn AD, De Ferrari GV and Kaykas A: Wnt and beta-catenin signalling: Diseases and therapies. Nat Rev Genet 5(9): 691-701, 2004.

4 Larue L and Delmas V: The wnt/beta-catenin pathway in melanoma. Front Biosci 11: 733-742, 2006.

5 Schepsky A, Bruser K, Gunnarsson GJ, Goodall J, Hallsson JH, Goding CR, Steingrimsson E and Hecht A: The microphthalmiaassociated transcription factor mitf interacts with beta-catenin to determine target gene expression. Mol Cell Biol 26(23): 8914$8927,2006$.

6 Hartman ML and Czyz M: Mitf in melanoma: Mechanisms behind its expression and activity. Cell Mol Life Sci 72(7): 12491260, 2015.

7 Garraway LA, Widlund HR, Rubin MA, Getz G, Berger AJ, Ramaswamy S, Beroukhim R, Milner DA, Granter SR, Du J, Lee C, Wagner SN, Li C, Golub TR, Rimm DL, Meyerson ML, Fisher DE and Sellers WR: Integrative genomic analyses identify mitf as a lineage survival oncogene amplified in malignant melanoma. Nature 436(7047): 117-122, 2005.

8 Goswami S, Tarapore RS, Poenitzsch Strong AM, TeSlaa JJ, Grinblat Y, Setaluri V and Spiegelman VS: Microrna-340mediated degradation of microphthalmia-associated transcription factor (mitf) mrna is inhibited by coding region determinantbinding protein (crd-bp). J Biol Chem 290(1): 384-395, 2015.

9 Bell JL, Wachter K, Muhleck B, Pazaitis N, Kohn M, Lederer M and Huttelmaier S: Insulin-like growth factor 2 mrna-binding proteins (igf2bps): Post-transcriptional drivers of cancer progression? Cell Mol Life Sci 70(15): 2657-2675, 2013.

10 Mahaira LG, Katsara O, Pappou E, Iliopoulou EG, Fortis S, Antsaklis A, Fotinopoulos P, Baxevanis CN, Papamichail M and Perez SA: Igf2bp1 expression in human mesenchymal stem cells significantly affects their proliferation and is under the epigenetic control of tet $1 / 2$ demethylases. Stem Cells Dev 23(20): 25012512, 2014.

11 Dietrich A, Tanczos E, Vanscheidt W, Schopf E and Simon JC: High cd44 surface expression on primary tumours of malignant melanoma correlates with increased metastatic risk and reduced survival. Eur J Cancer 33(6): 926-930, 1997.

12 King DT, Barnes M, Thomsen D and Lee CH: Assessing specific oligonucleotides and small molecule antibiotics for the ability to inhibit the crd-bp-cd44 rna interaction. PLoS One 9(3): e91585, 2014.

13 Craig EA and Spiegelman VS: Inhibition of coding region determinant binding protein sensitizes melanoma cells to chemotherapeutic agents. Pigment Cell Melanoma Res 25(1): 8387, 2012.

14 Utikal J, Leiter U, Udart M, Kaskel P, Peter RU and Krahn GM: Expression of c-myc and bcl-2 in primary and advanced cutaneous melanoma. Cancer Invest 20(7-8): 914-921, 2002.
15 Perry AM, Alvarado-Bernal Y, Laurini JA, Smith LM, Slack GW, Tan KL, Sehn LH, Fu K, Aoun P, Greiner TC, Chan WC, Bierman PJ, Bociek RG, Armitage JO, Vose JM, Gascoyne RD and Weisenburger DD: Myc and bcl2 protein expression predicts survival in patients with diffuse large b-cell lymphoma treated with rituximab. Br J Haematol 165(3): 382-391, 2014.

16 Busam KJ and Jungbluth AA: Melan-a, a new melanocytic differentiation marker. Adv Anat Pathol 6(1): 12-18, 1999.

17 Du J, Miller AJ, Widlund HR, Horstmann MA, Ramaswamy S and Fisher DE: Mlana/mart1 and silv/pmel17/gp100 are transcriptionally regulated by mitf in melanocytes and melanoma. Am J Pathol 163(1): 333-343, 2003.

18 Griewank KG: Biomarkers in melanoma. Scand J Clin Lab Invest Suppl 245: S104-112, 2016.

19 Ploper D, Taelman VF, Robert L, Perez BS, Titz B, Chen HW, Graeber TG, von Euw E, Ribas A and De Robertis EM: Mitf drives endolysosomal biogenesis and potentiates wnt signaling in melanoma cells. Proc Natl Acad Sci USA 112(5): E420-429, 2015.

20 Lin YT and Chao CC: Identification of the beta-catenin/jnk/ prothymosin-alpha axis as a novel target of sorafenib in hepatocellular carcinoma cells. Oncotarget 6(36): 38999-39017, 2015.

21 Noubissi FK, Elcheva I, Bhatia N, Shakoori A, Ougolkov A, Liu J, Minamoto T, Ross J, Fuchs SY and Spiegelman VS: Crd-bp mediates stabilization of betatrcp1 and c-myc mrna in response to beta-catenin signalling. Nature 441(7095): 898-901, 2006.

$22 \mathrm{Hu} \mathrm{MC}$ and Rosenblum ND: Smad1, beta-catenin and tcf4 associate in a molecular complex with the myc promoter in dysplastic renal tissue and cooperate to control myc transcription. Development 132(1): 215-225, 2005.

23 Jiang X, Kim HE, Shu H, Zhao Y, Zhang H, Kofron J, Donnelly J, Burns D, Ng SC, Rosenberg S and Wang X: Distinctive roles of phap proteins and prothymosin-alpha in a death regulatory pathway. Science 299(5604): 223-226, 2003.

24 Zhang M, Cui F, Lu S, Lu H, Jiang T, Chen J, Zhang X, Jin Y, Peng $\mathrm{Z}$ and Tang $\mathrm{H}$ : Increased expression of prothymosin-alpha, independently or combined with tp53, correlates with poor prognosis in colorectal cancer. Int J Clin Exp Pathol 7(8): 48674876, 2014.

25 Poste G, Doll J and Fidler IJ: Interactions among clonal subpopulations affect stability of the metastatic phenotype in polyclonal populations of b16 melanoma cells. Proc Natl Acad Sci USA 78(10): 6226-6230, 1981.

26 Katsara O, Mahaira LG, Iliopoulou EG, Moustaki A, Antsaklis A, Loutradis D, Stefanidis K, Baxevanis CN, Papamichail M and Perez SA: Effects of donor age, gender, and in vitro cellular aging on the phenotypic, functional, and molecular characteristics of mouse bone marrow-derived mesenchymal stem cells. Stem Cells Dev 20(9): 1549-1561, 2011.

27 Takemoto CM, Yoon YJ and Fisher DE: The identification and functional characterization of a novel mast cell isoform of the microphthalmia-associated transcription factor. J Biol Chem 277(33): 30244-30252, 2002. 